\title{
Feux conjugués ou opposés
}

Le dialogue entre lettres et sciences à la lueur des notes de Hermann pour L'Homme des champs de Delille

Friend or Foe. The dialogue between literature and science in the light of

Hermann's notes for Delille's L'Homme des champs

\section{Hugues Marchal}

\section{(2) OpenEdition \\ Journals}

Édition électronique

URL : http://journals.openedition.org/aes/2826

DOI : 10.4000/aes.2826

ISSN : 2258-093X

Éditeur

Laboratoire LISAA

Référence électronique

Hugues Marchal, «Feux conjugués ou opposés », Arts et Savoirs [En ligne], 13| 2020, mis en ligne le 01 juin 2020, consulté le 16 juin 2020. URL : http://journals.openedition.org/aes/2826 ; DOI : https:// doi.org/10.4000/aes. 2826

Ce document a été généré automatiquement le 16 juin 2020.

Centre de recherche LISAA (Littératures SAvoirs et Arts) 


\section{Feux conjugués ou opposés}

Le dialogue entre lettres et sciences à la lueur des notes de Hermann pour L'Homme des champs de Delille

Friend or Foe. The dialogue between literature and science in the light of

Hermann's notes for Delille's L'Homme des champs

Hugues Marchal

1 Poème descriptif et didactique publié en 1800, L'Homme des champs de Jacques Delille apparait comme la réalisation tardive de plusieurs idéaux des Lumières ${ }^{1}$. Dans la lignée de Rousseau et comme nombre d'auteurs du second dix-huitième siècle, l'abbé Delille conseille à ses lecteurs de chercher le bonheur à la campagne ${ }^{2}$. Les quatre chants qui forment son poème leur proposent successivement d'exercer la bienfaisance autour de leurs domaines, de contribuer au développement économique grâce à de grands travaux inspirés par l'agronomie moderne, de s'initier aux sciences naturelles pour augmenter leurs jouissances face au monde environnant et, enfin, de se faire euxmêmes artistes, en apprenant à peindre en vers ces sites. Le thème des deux chants centraux fait de ce texte, sinon un manuel de vulgarisation, du moins un ouvrage destiné à promouvoir un intérêt pour les sciences et les techniques ${ }^{3}$. Pour ce faire, Delille, qui a travaillé à ce poème dès les années 1770, a étroitement dialogué avec différents savants. Non seulement il a soumis ses vers, à différents stades de leur composition, à l'approbation de scientifiques", mais le texte imprimé s'accompagne d'abondantes notes explicatives en prose qui furent rédigées, pour la partie scientifique, par le naturaliste strasbourgeois Jean Hermann. Enfin, aborder en alexandrins les sciences contemporaines obéit aussi à une volonté de renouveler la poésie. D'une part, l'enjeu est générique : peindre les savoirs permet de contester les hiérarchies esthétiques traditionnelles, car chez Delille, le recours au poème didactique et descriptif, réputé aride et inférieur à l'épopée, à la tragédie ou au lyrisme usuel, se présente explicitement comme une tentative pour forcer lecteurs et critiques à remettre en cause la validité d'un tel classement ${ }^{5}$. D'autre part, l'enjeu est thématique : le tropisme vers les sciences illustre un désir de moderniser et d'élargir les motifs considérés comme poétiques. En effet, lorsque Delille juge dans sa préface que, dans L'Homme des champs, l'histoire naturelle lui a offert le sujet «le plus fécond de tous, et 
[que] jamais une carrière et plus vaste et plus neuve ne fut ouverte à la poésie » (HDC, p. xxx), il rejoint certaines propositions de Marmontel ${ }^{6}$ ou d'André Chénier qui avait déjà proclamé, dans L'Invention :

Torricelli, Newton, Kepler et Galilée, [...]

À tout nouveau Virgile ont ouvert des trésors.

Tous les arts sont unis : les sciences humaines

N'ont pu de leur empire étendre les domaines,

Sans agrandir aussi la carrière des vers. ${ }^{7}$

On le voit, bien des éléments incitent à aborder la partie scientifique de L'Homme des champs comme la mise en œuvre d'un programme qu'allégorise le frontispice de l' Encyclopédie. De même que dans la gravure de Cochin [fig. 1], poésie et science unissent leurs forces au service du rayonnement de la vérité. La poésie apporte aux sciences sa forme plaisante et sa capacité à séduire, tandis que ces dernières lui fournissent une matière lui permettant de briller de nouveaux feux, en une relation dont l'harmonie est encore soulignée par le fait que, si le poète se présente comme le lecteur des savants, le savant qui annote ses vers se place face à eux dans une position identique.

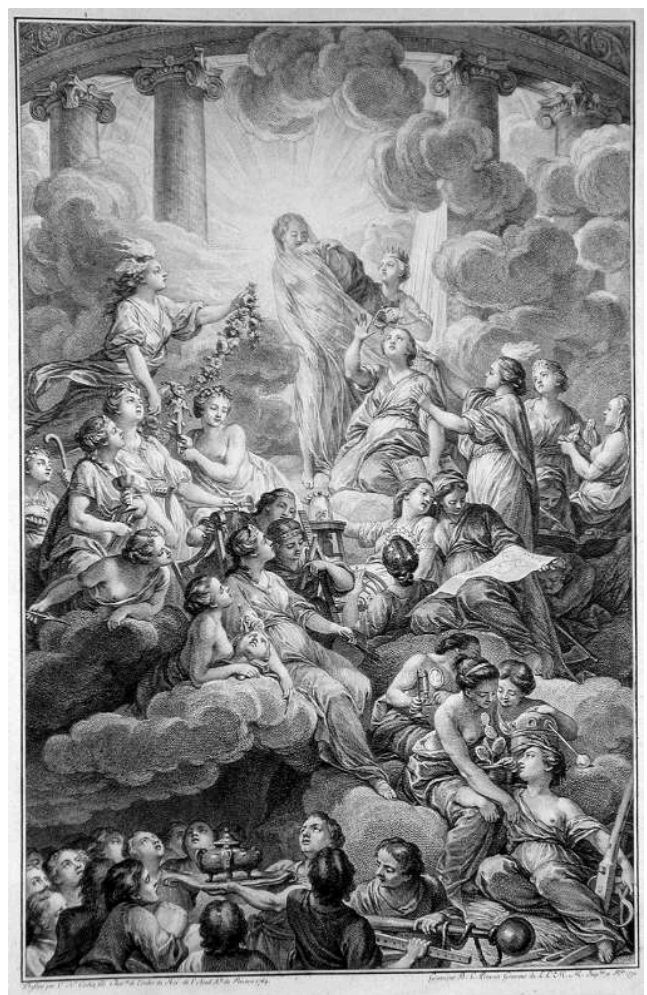

Fig. 1. Frontispice de Cochin pour l'Encyclopédie (1764, gravé par Prévost, 1769), coll. part. Tandis qu'au-dessus des sciences, la raison et la philosophie (à droite) dévoilent la vérité, l'imagination (à gauche) s'élance pour la couronner et l'embellir de fleurs, au-dessus des différentes formes de poésie et d'art.

Or il semble que, pour ses contemporains, Delille soit parvenu magistralement à accomplir ce programme. L'Homme des champs s'arracha à des milliers d'exemplaires dès les premières semaines et fit l'objet de nombreuses traductions, ainsi que d'une édition augmentée, en 1805. La majeure partie de la critique, malgré des réserves sur le poème, salua le troisième chant comme un chef-d'œuvre, aussi réussi que neuf. Enfin, le poème fut suivi, en 1808, d'une œuvre plus nettement encore liée aux sciences, Les Trois Règnes de la nature, cette fois annotée par des savants de tout premier plan, dont Cuvier. Ces deux œuvres peuvent donc apparaître comme l'une des manifestations les plus 
éclatantes du modèle culturel si fréquemment associé aux Lumières, celui d'une République fédérant gens de science et gens de lettres - au sens actuel de ce dernier terme - alors même que ces poèmes ont paru à un moment où, sous la plume de Chateaubriand ou Bonald, les premiers signes de scission apparaissaient, qui allaient imposer au XIX ${ }^{\mathrm{e}}$ siècle le motif obsédant d'une guerre à mort menée par les savants contre la poésie ${ }^{8}$.

Dans le cas de L'Homme des champs, l'hypothèse est d'autant plus séduisante qu'au moment où l'ouvrage paraît, Delille, né en 1738 et mort en 1813, et Hermann, né la même année et mort en octobre 1800, pouvaient l'un et l'autre se ranger parmi les derniers témoins de ce vaste mouvement.

5 Toutefois, L'Homme des champs doit-il vraiment être lu comme la trace fiable d'une collaboration heureuse entre poésie et science, invitant le lecteur moderne à regretter une sorte de paradis dialogique perdu? Ne faut-il pas plutôt traiter l'œuvre, à l'instar du frontispice de l'Encyclopédie, comme une représentation idéalisée de ce commerce? Un détail crucial incite à poser ces questions. Contrairement aux Trois Règnes, où le nom de Cuvier apparait dès la page de titre, L'Homme des champs fut publié sans aucune mention de la contribution de Hermann. Pourtant, ce savant respecté disposait d'un solide crédit: médecin et naturaliste, professeur de botanique à l'université de Strasbourg, il avait réuni un cabinet de curiosités et une bibliothèque très admirés ${ }^{9}$ et il avait, comme Delille, fait l'objet des attentions de la Convention ${ }^{10}$. On pourrait donc à bon droit être surpris que le poète ou ses éditeurs n'aient pas saisi l'opportunité de mettre en valeur la caution qu'il apportait au texte, si les archives de Hermann ne contenaient un ensemble de pièces relatives à L'Homme des champs, qui révèlent le caractère houleux de la collaboration.

6 C'est sur ces pages, à bien des égards exceptionnelles, car on ne dispose d'aucun vestige similaire pour étudier la genèse de l'annotation des Trois règnes ou d'autres textes du même type, que je me concentrerai, en présentant brièvement les manuscrits, puis en montrant que Hermann y apparaît sous trois visages distincts : celui d'un collaborateur au sens fort du terme, qui entend fondre sa voix et ses apports dans une œuvre commune ; celui, au contraire, d'un expert humilié, dont les conseils sont ignorés ; celui, enfin, d'un opposant, qui saperait de l'intérieur le projet même de L'Homme des champs.

\section{Les manuscrits de Strasbourg}

7 La publication de L'Homme des champs est étroitement liée à l'émigration de Delille, qui quitta la France pour la Suisse fin 1796. C'est à cette date qu'il accepte de vendre son manuscrit à deux éditeurs, le Strasbourgeois Levrault et le Bâlois Decker ${ }^{11}$. Or dès décembre, ce dernier annonce à son associé français que le poète, arrivé deux mois plus tôt à Bâle, «a fini son poeme ». Decker, enthousiaste, juge alors le texte plus beau encore que les Jardins, dernier poème en date de Delille, paru en 1782, et il ajoute : « il ne s'agit plus que des notes ${ }^{12}$. Deux mois plus tard, une lettre de février 1797 indique que c'est Levrault qui s'est chargé de rassembler cet appareil critique : Decker ironise en effet sur les problèmes que les "grands savans" consultés à Strasbourg par son partenaire rencontrent pour commenter certains vers de Delille ${ }^{13}$. Enfin, en juillet, Decker s'interroge sur la qualité du travail de Hermann, dont le nom apparait pour la première fois dans cette correspondance, ce qui permet de faire débuter les contributions de ce dernier, a minima, au printemps $1797^{14}$. Mais la tâche s'éternise. Un 
an plus tard, Decker confronte Levrault à ses retards : « Il y a 4 mois que vous disiér avoir les notes de Hermann. D'où vient qu'aujourd'hui vous ne les avér pas? $»^{15} \mathrm{Et}$ les premiers volumes ne seront mis en vente qu'en août 1800, peu avant la mort du naturaliste ${ }^{16}$. On doit donc dater le travail de Hermann entre le début de l'année 1797 et celui de cette dernière année.

8 Les documents manuscrits relatifs à cette tâche, conservés à la Bibliothèque nationale et universitaire de Strasbourg, se déploient sur 53 folios, classés après la mort du naturaliste par son frère, le juriste et homme politique Jean-Frédéric Hermann ${ }^{17}$. Ces pièces ne sont pas un état final des notes : ce sont des navettes intermédiaires.

9 La première étape du travail, du moins l'état le plus ancien conservé, consiste pour Hermann à parcourir la copie qu'il a reçue. Il signale les passages où il soupçonne " quelqu'erreur » ou une " faute de copiste » ( $f^{\circ} 365 \mathrm{r}$ ). En outre, il recense les vers pour lesquels une note paraît souhaitable ou non, sans qu'on puisse déterminer si une première liste préalable lui avait été communiquée à cette fin ou s'il cherche à établir cet inventaire avec ses interlocuteurs, avant d'entamer sa rédaction.

Les autres pièces du dossier enregistrent différents échanges successifs entre le naturaliste et un ou plusieurs des responsables de l'édition. Ils suggèrent des corrections à ses propositions de notes rédigées, en ajoutant leurs commentaires en marge ou en amendant directement son texte. Le dernier état envoyé par Hermann manque vraisemblablement, puisqu'il aura été conservé par les imprimeurs-libraires.

11 Les liasses ne comportent aucune référence aux chants I et IV, ce qui tend à suggérer que Hermann n'a pas eu accès à ces derniers. Il ne semble pas non plus avoir pu correspondre directement avec Delille, qui du reste quitta Bâle dès le milieu de l'année 1797. Les manuscrits n'évoquent jamais le poète qu'à la troisième personne, y compris quand Hermann soumet une demande de précision à laquelle seul l'auteur pourra répondre. Ainsi, lorsqu'il hésite à reconnaître l'araignée dans l'insecte que Delille nomme « tapissière agile $»^{18}$, il indique : «Le poëte seroit à consulter par écrit, \& alors on mettroit tout simplement, que c'est tel ou tel insecte qu'il entend » ( $\left.f^{\circ} 400 r\right)$.

\section{Une voix concordante}

12 À certains égards, les notes de Hermann témoignent d'un dialogue heureux. Elles archivent une lecture approbatrice et un désir de contribuer harmonieusement à la diffusion du poème.

13 Puisqu'il a accepté de rédiger ces notes, on peut penser que Hermann adhérait d'emblée au projet de Delille. De fait, il semble avoir partagé l'un des arguments clés du poète. Delille associe bonheur et sciences naturelles en présentant ces dernières comme un principe de démultiplication des plaisirs offerts au contemplateur du monde. Le début du chant III l'expose clairement, en insistant sur la notion de jouissance :

QUE j'aime le mortel, noble dans ses penchants, Qui cultive à la fois son esprit et ses champs !

Lui seul jouit de tout. Dans sa triste ignorance

Le vulgaire voit tout avec indifférence :

Des desseins du grand Être atteignant la hauteur,

Il ne sait point monter de l'ouvrage à l'auteur. [...]

Cherchez autour de vous de riches connoissances

Qui, charmant vos loisirs, doublent vos jouissances. 
Trois règnes à vos yeux étalent leurs secrets.

Un maître doit toujours connoître ses sujets :

Observez les trésors que la nature assemble.

Venez; marchons, voyons, et jouissons ensemble. ${ }^{19}$

Or les archives de Hermann contiennent un folio
notes, où une idée assez similaire est exposée :

L'homme peut, et il doit, jouir des dons de la Nature, des productions du globe qu'il habite.

Cette jouissance ne consiste pas seulement, en ce qu'il [...] les employe pour ses besoins soit essentiels soit factices, mais aussi en ce qu'il apprend à connoître les choses qui ne lui sont pas d'une utilité immédiate, \& qu'il admire la magnificence \& la variété de la Nature, la Sagesse et la Pompe - puissance du créateur.

[Les sciences appliquées] lui apprennent à se servir avec le plus d'avantage des productions de la nature [mais il est aussi une] science qui nous fait jouir de la nature uniquement en contemplant la beauté, la multiplicité, la variété, l'harmonie de tous les objets ${ }^{20}[\ldots .$.$] .$

s'applique en effet à dessiner un espace commun aux poètes et aux savants. À ce titre, il lui arrive de traiter Delille comme un naturaliste habile, dont il salue la finesse des compétences scientifiques. Ainsi, lorsque, dans un segment célébrant l'immense variété des animaux, le poète invite son lecteur à observer «Le poil uni du rat, les dards du hérisson $~_{22}$, Hermann approuve ce choix en indiquant : "l'extrême dans les poils est bien choisi ; car [les piquants] sont de véritables poils » (fo 394v). Inversement, le savant se place lui-même sur le terrain de l'homme de lettres, pour soumettre à celui qu'il nomme à plusieurs reprises le bon Delille des observations esthétiques ou linguistiques. Il repère avec acuité les erreurs qui émaillent la copie dont il dispose et souvent, il restitue avec justesse les termes corrompus. Tout en se plaignant que les éditeurs puissent jouer ainsi, par manque de rigueur dans leur relecture, un mauvais tour au poète qui était à cette date presque aveugle, il manifeste son souci d'aider l'écrivain à atteindre la perfection. C'est dans le même esprit qu'il signale, par exemple, des répétitions ou tournures qu'il « croi[t] que Mr. de L['Isle] voudra [...] éviter » (f' 368v).

De plus, Hermann réagit fréquemment en bon lecteur de cette écriture particulière qu'est la poésie. Il se montre attentif au contexte lorsqu'il doit hasarder une interprétation. Face à la formule « l'animal [...] dont la coquille est arrondie en voûte » ${ }^{23}$, il remarque : «Il ne peut guère être entendu d'autre animal que l'armadille ou le tatu, dont il y a plusieurs espèces en Amérique : et la tortue étant déjà nommée tout-àl'heure " (f॰ 393r). De fait, la périphrase qui retient son intérêt s'inscrit dans une liste où Delille vient d'évoquer " la lente tortue ${ }^{24}$; il a donc raison de chercher le référent de cette expression dans un autre animal. Pourtant, le volume imprimé transformera sa proposition en "C'est la tortue ou le tatou » ${ }^{25}$, faussant ainsi l'interprétation du savant, comme sans doute le sens du texte. Quand au contraire Hermann juge un tour polysémique, il tend à offrir en note une liste de possibilités. Là où Delille parle des insectes «dont l'amour dans l'ombre allume le flambeau " ${ }^{26}$, son glosateur évoque les abeilles ou les termites, dont on n'avait pu encore observer l'accouplement ; mais aussi les carabes et d'autres coléoptères «dont l'accouplement se fait ordinairement à couvert» (f०400v), autant de précisions cette fois conservées dans la version imprimée ${ }^{27}$. 
D'autre part, Hermann s'applique à éviter le pédantisme, crime fatal dans le régime discursif que Delille cherche à établir. Comme ce dernier l'expliquera plus tard, l'homme du bon ton, à la conversation aimable, doit s'astreindre à censurer son savoir. Fuyant dans ses discours « également la morgue du docteur, / Et du savant dissertateur / La prolixe pédanterie $»^{28}$,

Il ne se venge pas sur nous de ses travaux, Ne nous punit point de ses veilles : [...]

En légères vapeurs sa science s'exhale,

Se laisse deviner, et jamais ne s'étale

Dans des discours ambitieux. [...]

Sans effort, sans bruit, sans fracas,

Son savoir se répand et ne déborde pas. ${ }^{29}$

19 Hermann minore donc à plusieurs reprises son autorité, laissant aux éditeurs une grande latitude dans le traitement de ses propositions. Ainsi, quand il se rend compte qu'il a par inadvertance composé deux notes différentes pour un même passage, il indique : « on choisira, ou fondra en une seule » (f॰ 391r). C'est qu'il est conscient de la nécessité d'éviter des développements trop amples. Le poème n'ayant pas vocation à se substituer à un manuel savant et devant, au contraire, dans la tradition lucrétienne, masquer les leçons qu'il propose sous une apparence de facilité, le naturaliste fournit à plusieurs reprises des notes très brèves, qui ont trouvé place dans le volume définitif sans aucune modification ${ }^{30}$. Si Hermann craint qu'un terme exige une note trop complexe, sans qu'une glose ne s'impose pour éclairer le sens des vers, il préfère donc renoncer à tout commentaire. Ainsi, lorsque Delille mentionne les "trois règnes », le naturaliste commence par écarter l'éventualité d'une note, en demandant: «S'attendra-t-on ici à une définition des trois règnes? On auroit l'air de vouloir endoctriner fort mal à propos » ( $f^{\circ} 365 \mathrm{r}$ ). Ailleurs, il motive ce refus d'annoter par le fait que le discours du poète heurte ses propres connaissances. Plutôt que de se hasarder à rédiger une remarque discordante, il opte pour un silence dont il se contente d'avertir ses interlocuteurs. C'est le cas lorsqu'au début du chant III, Delille plaint l'ignorant,

[Qui] ne sait pas comment, dans ses secrets canaux,

De la racine au tronc, du tronc jusqu'aux rameaux,

Des rameaux au feuillage accourt la sève errante ${ }^{31}[. .$.$] .$

Hermann indique :

On ne voudra pas que je mette ici une note explicative. On ne s'attendra pas à ce que je concentre dans un petit éspace le resultat des recherches \& des expériences de Grew, Malpighi, Duhamel, Hill, Desfontaines, \&c. \& que je décide entre les différens sentimens. Surtout parceque moi je suis d'avis que ce sont les feuilles surtout qui pompent les principes de l'atmosphère \& qui les envoient aux rameaux, $\&$ de la au tronc, par un chemin contraire à celui qu'adopte Mr. de l'Isle. (f' $365 r$ )

21 On comprend la difficulté. Ici, le naturaliste juge manquer d'espace pour exposer l'état des savoirs; il signale qu'il serait obligé d'adopter une thèse divergeant de celle du poète et surtout, il indique que l'exemple utilisé par Delille est mal choisi, la circulation de la sève ne constituant en aucun cas un "savoir " ferme, dont les amateurs de sciences naturelles jouiraient. Mais puisque la position du poète rejoint celle d'autres botanistes, Hermann décide de passer la difficulté sous silence et de renoncer à une dissertation complexe.

Enfin, quand une note divergente semble inévitable, il arrive que Hermann explore des solutions respectant le discours du poète. Il cherche une parole de conciliation, un 
compromis possible. Par exemple, le naturaliste propose de compléter l'expression « les dards du hérisson $»^{32}$, qu'il juge susceptible d'induire le lecteur en erreur, par une note distinguant exactitude savante et justesse esthétique. La formule est acceptable à condition de ne pas être comprise au sens littéral :

Cette expression est poëtique. Il ne faut pas qu'elle fasse croire que le hérisson darde ses piquants comme on l'avoit prétendu [du] porc-épic. Ni l'un ni l'autre de ces animaux n'ont la faculté de lancer comme une arme offensive les aiguillons dont ils sont couverts. (f॰ $394 r)$

Il en va de même lorsque Hermann est confronté à un passage où Delille indique que, sous nos climats, certains animaux, comme le tigre ou le lion, ne parviennent plus à se reproduire. Plusieurs exemples d'élevage réussi infirmant cette thèse, Hermann débute sa proposition de note par la formule : «Après avoir rapporté l'opinion énoncée dans les vers par des autorités respectables, nous croyons faire plaisir aux amateurs des sciences naturelles \& rassembler quelques faits qui sont en contradiction avec eux " ( $f^{\circ} 361 r$ ). On le voit, non seulement Hermann indique à nouveau que les déclarations de Delille, qu'il récuse, peuvent néanmoins trouver un écho chez certains de ses pairs, mais la phrase brouille la distinction entre le poète et son correcteur, en les réunissant dans un même "nous", présenté comme l'instance responsable d'une ventilation volontaire des éléments fournis au lecteur.

Ce travail de conciliation peut impliquer de fastidieux va-et-vient. Dès son premier parcours du chant III, Hermann s'arrête sur un passage où Delille, évoquant la longue histoire géologique du globe, en donne pour preuve l'existence de bois fossilisés, qu'il désigne par le tour : « ces grands amas dans la terre enfermés, / Ces bois, noirs aliments des volcans enflammés $»^{33}$. Hermann signale sa perplexité face au dernier vers, en notant: "Je ne crois pas qu'aucun auteur ait attribué les volcans à des bois en combustion, \& son opinion ne seroit pas admissible» (f $\left.{ }^{\circ} 365 \mathrm{v}\right)$. En d'autres termes, Hermann suggère à Delille de réécrire le passage pour en ôter la formule problématique - et ce type de suggestion n'est pas isolé, on le verra. Or une main tierce a ajouté, en marge de cette première remarque: "laissez le bois ». Dans la navette suivante, Hermann revient donc sur le passage et en donne un commentaire qui débute par une adresse directe :

Que d'autres décident, si cette expression peut être passée à un poëte, surtout dans un genre qui approche si fort du didactique, où pour ne pas donner lieu à des erreurs, les termes doivent être aussi mesurés, <aussi éloignés du figuré,> \& exciter des idées aussi vraies que possible. Il est certain qu'il y a des bois souterrains, des forêts entières, où les buches ou du moins les troncs sont encore des plus distincts. $<$ Tels sont les charbons [de bois].> Mais ce n'est pas là ce qui nourrit les volcans [...] que ce soyent même les pyrites [...] qui nourrissent le feu des volcans, c'est là une chose sur laquelle tous les naturalistes ne sont pas encore d'accord. (fo $371 \mathrm{v}-372 \mathrm{r}$ )

Hermann rend ici explicite une tension qui demeurait implicite dans le cas du hérisson: le poète didactique doit s'interdire certaines ambivalences typiques de la poésie, dès lors qu'elles risquent d'induire le lecteur en erreur. C'est pourquoi l'annotateur refuse de valider la figure. Mais Hermann ne limite pas sa critique à cette remarque. Il continue en évoquant les travaux de Spallanzani et d'autres chercheurs, avant d'indiquer qu'il existe un «système assez généralement reçu sur l'aliment des volcans » et que « le poëte pouvoit fort bien l'adopter de même » (fo $372 \mathrm{v})$. Il reproche donc aussi à Delille d'exposer une vue minoritaire, voire absente de tout ouvrage scientifique (ce qu'il avait signalé dès sa première remarque en notant qu'« aucun auteur » n'avait exprimé la thèse adoptée par Delille). Puis le naturaliste entreprend de 
justifier longuement sa position, en passant en revue différentes expériences menées notamment par Lemery et en discutant de leurs résultats, avant de conclure que la question "reste une des plus problématiques » ( $\left.f^{\circ} 374 \mathrm{rv}\right)$. La note imprimée condense fortement cette dissertation scientifique et elle élimine, sans surprise, les deux remarques qui incriminent directement Delille. Elle s'applique néanmoins à déjouer le conflit potentiel entre poésie et science, en exploitant la critique de Hermann sans l'adopter entièrement. La première phrase du texte imprimé, «On a voulu renfermer dans l'expression la plus succincte les différentes matières que la nature emploie pour l'entretien des feux volcaniques", prévient le lecteur que le mot rejeté par le naturaliste doit s'entendre comme une figure, en l'occurrence une métonymie, qui désignerait un ensemble de «matières » par une seule d'entre elles. La suite synthétise plusieurs des précisions scientifiques fournies par Hermann, pour informer que «les expériences de plusieurs physiciens célèbres », comme "Lemery, Homberg, Newton, Hoffman et Boerhaave ", " doivent au moins faire [douter] que le foyer des volcans soit alimenté par des végétaux fossiles $»^{34}$. En d'autres termes, la note finalement adoptée inscrit un désaccord potentiel (le mot employé à des fins métonymiques désigne une matière qui ne fait peut-être pas partie de l'ensemble qu'il doit représenter), mais la modalisation atténue le désaccord (il ne faut pas refuser l'idée, mais en douter) et la formule "on a voulu», par le choix d'un pronom indéfini, permet de suggérer que l'expression de Delille a été discutée et acceptée par le poète et l'instance en charge de l'annotation.

En somme, Hermann fournit des éléments dissonants que les éditeurs peuvent transformer en un matériel pour élaborer une note conciliatrice, respectant le vers et l'état des savoirs, quitte à recourir à des artifices rhétoriques. Or un tel dispositif permet de saisir tout l'intérêt du recours à un glosateur savant. Ici, en effet, c'est l'annexe en prose qui, tout en corrigeant la théorie véhiculée implicitement par le vers, lui permet de demeurer inchangé. Mutatis mutandis, la note installe un dialogisme comparable à celui qui structure aujourd'hui un entretien de vulgarisation: dans les deux cas, même si le savant corrige ou nuance certaines expressions de son interlocuteur, l'ensemble de l'échange reste un dispositif didactique efficace. Les tensions sont donc dépassées au profit d'une manifestation de concorde, comme si tous les acteurs s'appliquaient à produire le et pluribus unum des deux postures poétique et scientifique.

\section{Un collaborateur humilié}

Nombre des propositions de Hermann semblent toutefois avoir été radicalement ignorées.

On l'a vu, lorsqu'un vers lui pose un problème de vérité scientifique, il arrive que le Strasbourgeois suggère à Delille de procéder à une correction, qui résoudrait cette tension. Mais ces demandes ont été systématiquement écartées : sauf lorsque la copie remise au naturaliste présentait d'évidentes corruptions, les vers de Delille ont paru tels que Hermann les avait reçus, sans prise en compte de ses observations critiques.

Surtout, lorsque le naturaliste exprime un net désaccord, le conflit se résout rarement à la manière de l'exemple précédemment évoqué des volcans. La version imprimée règle beaucoup plus volontiers le problème en éliminant toute note face aux vers mis en cause. Et les réductions et oblitérations de la parole du savant ne se limitent pas à ces 
éléments. Lorsque Hermann suggère une note longue, la version finale n'en garde généralement que quelques lignes, gommant les nuances qu'il apportait, notamment sur le degré de certitude des connaissances ou sur la variété du vivant. Dans ce dernier cas, la polysémie des tournures du poète, telle que le savant l'avait mise en évidence, se trouve effacée. Par exemple, lorsque Delille évoque «la mouche qui file " ${ }^{35}$, Hermann commente : «l'auteur entend peut-être les mouches-à-soie ou les Tenthredo, ou bien la Formicaleo dont la larve file aussi [...] » (f॰ $400 \mathrm{v})$. En d'autres termes, Hermann propose une nouvelle fois une interprétation ouverte, permettant d'envisager deux référents ${ }^{36}$. Dans l'édition imprimée, la note devient pourtant : « Plusieurs naturalistes ont compris sous la dénomination de mouches les demoiselles dont les larves filent pour tapisser le logement où elles se métamorphosent [, comme le] formica leo $"^{37}$. Un seul des exemples proposés par le naturaliste est donc retenu. Or cette stratégie éditoriale a pu concourir à faire lire les périphrases de Delille comme des devinettes puériles, dont la solution toujours simple serait donnée en note, au lieu de montrer comment ses figures alternent précision et vague, renvoi à une espèce précise ou indication de catégories plus incertaines.

Enfin, l'édition imprimée efface tous les passages où le naturaliste dit «je », c'est-à-dire tous les moments où il fait état de ses propres recherches ou opinions ${ }^{38}$, et si l'un des buts avoués d'un poème comme L'Homme des champs est bien de permettre à la parole savante de se diffuser dans la société, l'une au moins de ces fins de non-recevoir paraît contredire de manière exemplaire un tel modèle.

31 À la fin du chant III, Delille conseille à ses lecteurs d'établir leur propre herbier ou collection d'histoire naturelle, mais il incite à écarter de ces cabinets les «os de géants ${ }^{39}$, c'est-à-dire les fossiles de grande taille, en arguant que de tels objets sont réservés aux cabinets spécialisés. Pour le savant strasbourgeois, il faudrait au contraire sensibiliser le grand public à l'importance de ces objets :

Moi, au lieu de dissuader le naturaliste campagnard de se fournir des os de géants, c. à. d. de grands animaux fossiles, je l'exciterois plutôt à mettre toute son attention à ce que, si des indices de pareils os se montrent, rien ne soit dégradé ni dispersé, mais que les fouilles se continuent avec soin \& intelligence, que toutes les circonstances \& les positions des os soyent observées. Combien de pareils objets des plus intéressans \& des plus précieux n'ont pas été abimés, parce qu'ils sont tombés dans des mains ignorantes ! (f॰ 404r)

Le début de la note est trop personnel ; il est donc biffé, jusqu'à « se montrent », tandis qu'une proposition de reformulation, d'une main difficile à identifier, apparaît en marge :

Moi, au lieu de dissuader le naturaliste campagnard de se fournir des os de géants, Ł.à.d. de grands animaux fossiles, je l'exciterois plutôt à mettre toute son attention à ce que, si des indices de pareils os se montrent, <L'on ne voudrait pas dire ici qu'il ne soit souvent intéressant de recueillir ce qui peut se découvrir de pareils objets ; ils appartiennent à l'histoire de la science. Lorsqu'il se montre des indices d'os de géants, co[mme] on les appelle, l'amateur doit mettre son attention à ce que> rien ne soit dégradé [...].

La note ainsi envisagée ne sera toutefois pas retenue, de sorte que le livre final écarte complètement le seul passage où Hermann a tenté d'exploiter la diffusion du poème pour sensibiliser le public à un enjeu majeur pour les sciences naturelles contemporaines, en l'occurrence la paléographie naissante. L'annotateur n'a pas eu voix au chapitre pour décider des messages à transmettre. 


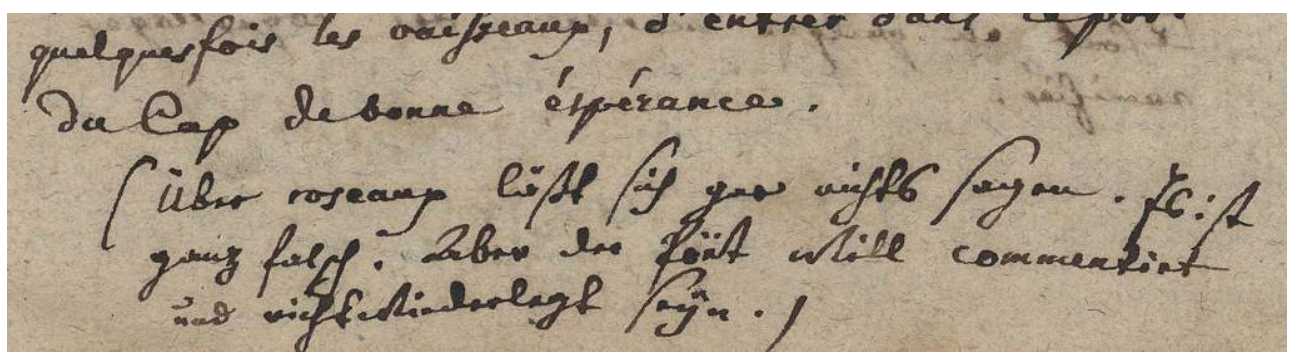

Fig. 2. Notes scientifiques, médicales, archéologiques et philosophiques, recueillies par Jean Hermann. Carton III, collection Bibliothèque nationale et universitaire de Strasbourg, cote MS.1.887, fo 381r, détail (photographie : Timothée Léchot). Hermann écrit entre parenthèses : «Über roseaux läßt sich gar nichts sagen. Es ist ganz falsch. Aber der Poët will commentiert und nicht wiederlagt seyn. » (Sur les roseaux on ne peut rien dire. C'est entièrement faux. Mais le poète veut être commenté, et non réfuté).

Détail singulier, le naturaliste emploie l'allemand en quelques rares occurrences, pour n'être compris que des éditeurs, et non de Delille, qui ne parlait pas cette langue. Or il se plaint, dans un de ces passages, de ce que «le poète veut être commenté, et non réfuté » [fig. 2]. Hermann considère donc que le poète, par son obstination, est le principal responsable des difficultés qu'il éprouve ou, du moins, il fait ponctuellement mine d'adopter cette posture, pour inviter les éditeurs à davantage prendre en compte ses remarques, sans les attaquer directement. Mais ces derniers semblent avoir traité les développements du naturaliste comme une forme de cuistrerie rompant le pacte d'écriture attendu. Decker, quand il nomme pour la première fois Hermann, n'en parle à Levrault que pour lui suggérer de s'en débarrasser :

Si à la place du pédant hermann Vous trouviér un jeune homme instruit et sachant le françois, auquel on marqueroit les passages pour lesquels il faut des notes, pour qu'il en fasse d'après un bon dictionnaire d'histoire naturelle, je crois que cela vaudroit mieux ${ }^{40}$.

Si le savant établi peut sembler ici injustement traité, il n'avait guère facilité la tâche à ses interlocuteurs, car il se comporte souvent en agresseur.

\section{Un partenaire difficile}

Certes, les refus que le Strasbourgeois essuie, lorsqu'il s'exprime en tant que naturaliste, suggèrent que son expertise n'est pas respectée par ses interlocuteurs. Mais leur désinvolture a pour pendant sa propre propension à mettre en cause l'autorité poétique de Delille. Certaines des tensions les plus marquées se manifestent en effet sur un terrain où l'on aurait pu croire que le naturaliste se montrerait plus humble : celui de la correction esthétique et linguistique du texte.

Les erreurs du copiste gênent indéniablement la lecture de Hermann, qui n'est pas toujours en mesure de décider s'il a affaire aux mots de Delille ou à une version corrompue. Quoi qu'il en soit, et même s'il se montre, on l'a vu, souvent capable de restituer avec sagacité ce que «Delille doit avoir dicté» (f॰ $366 \mathrm{v}$ ), ses soupçons d'altération du texte révèlent les limites de l'expertise littéraire qu'il revendique. Face à des passages retors, mais dont on peut motiver la difficulté et valider la syntaxe, le glosateur tend systématiquement à résoudre ses incompréhensions en concluant de manière fort peu diplomatique à des fautes d'écriture, soit dans sa copie, soit chez le poète. Or il passe alors aisément de formules comme « Je lis, je relis, \& je ne trouve pas de liaison. Il manque quelque chose » à des remarques moins amènes, telles que : « C'est 
du galimathias [sic]. Il y a certainement des lacunes", ou: "Ce sont des pièces de morceaux » (fं $366 r)$. En outre, Hermann tend à rejeter les expressions hardies, au risque de leur substituer des clichés. Ainsi, là où Delille parle d'« Un sable infructueux, aux vents abandonné $»^{41}$, il s'insurge : « infructueux, au lieu d'infécond : les poëtes se le permettent-ils ? Le sable est stérile, la mère est inféconde, \& les travaux, les peines, les soins, sont infructueux » ( $\left.f^{\circ} 365 \mathrm{v}\right)$. En d'autres termes, le naturaliste tend à normaliser le poème avec un zèle de Zoïle, à partir d'un idéal de bien dire qui traite comme des maladresses des infractions très conscientes aux normes.

Les tensions sont toutefois aussi liées à des provocations directes de la part de Hermann. Car, à mesure que le travail avance et que ses propositions sont refusées ou reformulées, il semble, du moins dans certains folios, chercher à déclencher une guerre ouverte avec Delille et ses éditeurs.

Ce désir de heurter la sensibilité de ses interlocuteurs se manifeste dans des passages à teneur à la fois idéologique et satirique, dont on perçoit toute l'insolence si l'on se rappelle que l'abbé Delille est à la fois un clerc et, en tant qu'émigré, un proche de la Contre-Révolution. Pour s'en tenir ici encore à un seul exemple, lorsque Hermann rédige la note sur les "trois règnes" dont il avait d'abord écarté l'opportunité, il soumet une proposition de plus de cinq folios, au contenu nettement polémique. Dès les premières lignes, il critique l'usage du mot règne, qu'il juge désormais «déplacé en histoire naturelle " ( $\left.f^{\circ} 369 r\right)$, ce qui revient à proposer, dans la lignée de Daubenton, l'adoption d'un lexique conforme à l'esprit jacobin ${ }^{42}$. Puis, après avoir médité sur le fait que Linné aurait pu parler de "république » plutôt que d'« Empire de la nature », il fait mine de saluer le "sublime » des allégories du naturaliste suédois pour en proposer sa propre version, toute de provocation envers Delille. Si Linné avait parlé « tout-à-fait de notre goût ", explique Hermann, il aurait certainement

appelé le regne minéral le clergé, cet état ayant entassé les trésors de Plutus [...]. Il aurait appellé le regne animal la noblesse; les animaux carnassiers, destructeurs, consommateurs, lui auraient suggéré cette idée. Les végétaux enfin humbles en général \& foulés, mais les plus utiles en même tems des trois règnes \& les plus nombreux auroient figuré sous le nom de Tiers-état, et le passage du regne végétal à l'animal qui se trouve dans les polypes \& les zoophytes auroit servi de comparaison au roturier annobli. (f॰ $370 \mathrm{r}-371 \mathrm{v}$ )

Le savant termine ce morceau de bravoure en citant différents naturalistes modernes ayant par ailleurs proposé de distinguer plus de trois règnes. Mais, malgré ce retour à un ton sérieux, il est évident que Hermann conçoit cette proposition de note, dont la forme comme le contenu sont étrangers à son cahier des charges, comme une sorte de courrier interne, destiné à embarrasser et agacer ses partenaires. Le nous qu'il met en avant dans la formule « de notre goût » ne saurait être partagé avec Delille.

Pire, le naturaliste a recours à la menace pour tenter d'imposer certaines de ses exigences. Lorsque le poète, évoquant les plantes venues d'Orient que les jardiniers ont introduites sous nos climats, chante "L'arbre heureux de Judée, à la fleur purpurine »" Hermann soulève trois problèmes. D'une part, il repère une cheville: "Je ne saurais donner aucune explication à cette épithète d'heureux». D'autre part, il explique que l'appellation correcte, présente déjà chez De l'Écluse, est " arbre de Judas » et il précise qu'il n'a pu trouver la forme employée par Delille que dans la traduction française, évidemment fautive, d'un ouvrage du botaniste anglais Miller. Enfin, il juge ce nom de Judas lui-même « ridicule » et raille la «filiation d'idées » qui a conduit, selon lui, à associer cette plante, dont les feuilles ressemblent vaguement à des oreilles, à l'épisode 
du soldat à l'oreille coupée du Jardin des Oliviers et, de là, à "l'apôtre traitre " (fo 355v). En effet, Hermann explique que l'arbre, loin de venir du Levant, est natif de l'Europe méridional. Or le naturaliste, qui semble exprimer ici pour la première fois des réserves face au vers ${ }^{44}$, n'attend pas la réponse des éditeurs ou de Delille pour poursuivre sa rédaction. Il conclut cette remarque par une sommation :

C'est donc à savoir, si le poëte préfère

ou de perpétuer par de beaux vers une fausseté ridicule ;

ou permettre que j'y mette cette note ;

ou supprimer ces vers \& leur en substituer d'autres ;

ou, s'il ne veut faire ni l'un ni l'autre, s'y attendre que quelqu'un - peut-être moi même - lui donne sur les doigts <le critique> dans le Magazin [sic] encyclopédique ou ailleurs.

Il pourroit mettre quelqu'autre mot au lieu d'heureux, \& substituer Judas à Judée. (fo $356 \mathrm{r}$ )

En d'autres termes, Delille est sommé de s'exécuter. Opposant au chant un chantage, Hermann exige qu'il modifie son texte ou accepte d'être corrigé dans une note, faute de quoi il le menace de porter le conflit dans un des périodiques parisiens les plus influents, le Magasin encyclopédique de Millin, dont le titre se range précisément sous la bannière de l'Encyclopédie. Le ton est d'autant plus violent que, dans le passage barré mais demeuré lisible, le savant compare cette action à une punition réservée aux enfants. Quel impact cette fulmination a-t-elle eu sur l'édition imprimée? Le vers a bien paru comme tel : une nouvelle fois, les partenaires du naturaliste ont donc écarté sa demande de reformulation. En revanche, une brève note signale le problème terminologique, en s'appuyant sur les indications fournies par Hermann. Mais ses remarques sont concentrées de telle sorte que la glose présente le choix de Delille comme une licence, autorisée par le livre de Miller et par un prétendu usage « vulgai[re] » dont le savant strasbourgeois n'avait pour sa part nullement fait état :

La dénomination propre de cet arbre est celle d'arbre de Judas: la première, vulgairement adoptée en France, se trouve dans le Dictionnaire des jardiniers de Miller. De l'Écluse a dit, il y a deux cents ans, vulgus herbariorum arborem Judæ vocant ${ }^{45}$.

Surtout, l'erreur principale, rangeant l'arbre parmi les essences orientales, est entièrement passée sous silence.

On comprend dans ces conditions que Hermann ait eu le projet, après l'impression du texte, de mettre sa menace à exécution. Selon une lettre manuscrite adressée à Cuvier et conservée cette fois à l'Institut de France,

Les éditeurs [...] avaient demandé à Hermann des notes relatives aux objets d'histoire naturelle dont parlait Delille. Hermann en a fourni un bon nombre. Mais plusieurs de ces notes ont été ou tronquées, ou entièrement omises surtout celles qui contenaient des critiques et Hermann s'était proposé de faire insérer celles-ci dans quelque journal. La mort l'a empêché de les mettre au net ${ }^{46}$.

La collaboration entre Hermann et Delille apparaît donc comme une joute pleine de frustration pour le savant et d'inconfort pour le poète et ses éditeurs. Le dissensus est d'autant plus fort que le conflit implique une dimension épistémologique cruciale: dans des passages que j'ai écartés ici, Hermann défend en effet une science de l' expérimentation, qui s'oppose à la simple observation que le poème ne cesse de célébrer et par là, au statut générique même du texte, associant science et posture descriptive ${ }^{47}$. Pour rendre compte des difficultés éprouvées par le naturaliste, faut-il, comme Decker, incriminer son pédantisme, ou, comme Hermann, rendre Delille responsable des 
blocages? La réponse à cette question importe en réalité assez peu, car l'intérêt documentaire des manuscrits de Hermann est plus général. Ils permettent d'assister à la fabrique d'une représentation: comme le volume imprimé, l'image d'une union heureuse entre sciences et littérature, telle que les Lumières l'ont imposée, a sans doute souvent nécessité un travail complexe de réduction, voire de voilement des conflits qui pouvaient opposer les acteurs de ce dialogue - travail et contorsions que le poème scientifique voue usuellement à l'effacement, mais dont les manuscrits laissent entrevoir un exemple frappant ${ }^{48}$.

\section{NOTES}

1. Pour un panorama global de l'œuvre de Delille et de son contexte, voir Édouard Guitton, Jacques Delille (1738-1813) et le poème de la nature en France de 1750 à 1820, Paris, Klincksieck, 1974.

2. Il s'agit d'un lieu commun de la fin de l'Ancien Régime : en 1787-1788, Marie-Antoinette fait réaménager sa chambre du Petit Trianon en commandant un décor dit «au treillage » ou «aux épis ", conçu pour rappeler la vie champêtre et dont demeurent notamment deux bras de lumière réalisés par Thomire (voir João Castel-Branco Pereira (dir.), Musée Calouste Gulbenkian, Lisbonne, Fondation Calouste Gulbenkian, 2011, p. 169). Mais après 1789, les troubles révolutionnaires et le départ volontaire ou forcé d'une partie des élites loin de Paris donnent aussi à cette aspiration et à l'éloge de l'histoire naturelle un sens nouveau, reflété dans de nombreux poèmes didactiques contemporains. Tandis que le second chant de L'Homme des champs débute par l'exclamation: «HEUREUX qui dans le sein de ses dieux domestiques / Se dérobe au fracas des tempêtes publiques, / Et, dans un doux abri trompant tous les regards, / Cultive ses jardins, les vertus et les arts!» (Jacques Delille, L'Homme des champs, ou les Géorgiques françoises, Strasbourg, Levrault, an VIII-1800, p. 73 - référence désormais abrégée HDC), Lalanne présente les vers de son Potager en expliquant: "Je publie cet essai dans un temps où les jouissances du plus grand nombre des propriétaires, singulièrement diminuées par le malheur des circonstances, se trouvent resserrées dans des limites étroites telles que celles d'un potager. Puissent ces idées, jetées sans prétention sur le papier, leur faire trouver moins amer le souvenir de ce qu'ils ont perdu !» (Jean-Baptiste Lalanne, Le Potager, essai didactique, Paris, chez les marchands de nouveauté, an VIII-1800, p. 5).

3. Campenon, parmi d'autres, y insiste: «Un poëme n'est pas un traité. Pour qu'un poëme soit didactique, il suffit qu'il contienne quelques préceptes; et l'auteur, étant maître de les choisir, doit s'attacher de préférence à ceux qui font aimer l'art qu'il chante » (Vincent Campenon, La Maison des champs [1809], dans Poèmes et opuscules en vers et en prose, Paris, Ladvocat, 1823, tome II, p. 93).

4. Delille a bénéficié d'informations ou de conseils de lecture dispensés notamment par les chimistes Jean Darcet et Louis Lefèvre-Gineau, par les astronomes Jérôme de Lalande et JeanBaptiste Delambre, et par le naturaliste Eberhard August Wilhelm von Zimmermann.

5. Cette volonté d'ouvrir l'éventail des genres à succès est affirmée dès le début de la préface de L'Homme des champs : « la tragédie et la comédie, les romans, et les poésies nommées fugitives, ont long-temps exercé presque exclusivement tous nos poëtes ; les gens du monde, de leur côté, ne se sont guère occupés d'aucun autre genre de poésie [tandis que] nos voisins se glorifioient d'une foule de poëmes étrangers au théâtre et à la poésie légère » (HDC, p. II). 
6. Voir Hugues Marchal (dir.), Muses et ptérodactyles: la poésie de la science de Chénier à Rimbaud, Paris, Éditions du Seuil, 2013, p. 70.

7. André Chénier, «L'invention », Euvres complètes, éd. de G. Walter, Paris, Gallimard, coll. «Bibliothèque de la Pléiade », 1958, p. 125.

8. Voir Hugues Marchal (dir.), op. cit., p. 213-262.

9. Sur Hermann et son réseau savant, on consultera avec profit la thèse de doctorat en histoire moderne soutenue par Dorothée Rusque, Le Dialogue des objets. Fabrique et circulation des savoirs naturalistes : le cas des collections de Jean Hermann (1738-1800), Université de Strasbourg, 2018.

10. Le 14 nivôse an III (3 janvier 1795), sur proposition du Comité d'instruction publique, la Convention décrète des secours aux savants, gens de lettres et artistes «dont les talents honorent la partie » et sont "nécessaires" à la République. Delille et Hermann font partie des bénéficiaires de la gratification la plus élevée, 3000 livres (voir J. Guillaume (dir.), Procès verbaux du Comité d'instruction publique de la Convention nationale, Paris, Imprimerie nationale, 1904, tome V, p. 380-385).

11. Pour une présentation des deux firmes et de leurs rapports, voir Frédéric Barbier, Trois cents ans de librairie et d'imprimerie : Berger Levrault, 1676-1830, Genève, Librairie Droz, 1979.

12. Lettre de Georg Jakob II Decker à la maison Levrault, Bâle, 16 décembre 1796. Archives départementales du Bas-Rhin. Fonds Berger-Levrault, cote: 40J, sous-cote : 463, dossier 8, « Decker ».

13. Lettre de Decker à la maison Levrault, Bâle, 11 février 1797 , loc. cit.

14. Lettre de Decker à la maison Levrault, Bâle, 15 juillet 1797, loc. cit.

15. Lettre de Decker à la maison Levrault, Bâle, 23 juin 1798 , loc. cit.

16. Bien qu'elle ait fait l'objet de diverses annonces antérieures, la parution intervient à Paris le 20 août (voir [Jean-Gabriel Peltier], "L'Homme des champs [...]. Premier extrait », Paris pendant l'année $1800, \mathrm{n}^{\circ} \mathrm{CCX}, 30$ août 1800, p. 80, ainsi que Journal typographique et bibliographique, $3^{\mathrm{e}}$ année, $\mathrm{n}^{\circ} \mathrm{XLV}, 5$ fructidor an VIII [23 août 1800], p. 363-364). - Hermann meurt le 4 octobre.

17. L'ensemble forme une partie des Notes scientifiques, médicales, archéologiques et philosophiques, recueillies par Jean Hermann. Carton III, Bibliothèque nationale et universitaire de Strasbourg, cote MS.1.887. Les indications de folio $\left(f^{\circ}\right)$ qui suivent renvoient toutes à cette source; dans les transcriptions, les signes $<>$ encadrent les éléments ajoutés à une première rédaction sur le manuscrit même, tandis que les crochets droits ([]) signalent mes propres interventions.

18. HDC, p. 131.

19. HDC, p. 107-108.

20. $\mathrm{F}^{\circ} 179$ rv.

21. Ibid.

22. HDC, p. 130.

23. Id.

24. Id.

25. HDC, p. 242.

26. HDC, p. 131.

27. HDC, p. 246.

28. Jacques Delille, La Conversation, Paris, Michaud, 1812, p. 134.

29. Ibid., p. 137-138.

30. Par exemple, lorsque Delille parle des «poisons salutaires» des plantes (HDC, p. 125), Hermann propose: "Le médecin habile ne connoît guère de poison qui soit tel absolument. Employées prudemment \& à propos, les plantes réputées les plus venimeuses, la ciguë, la colchique, l'aconit, la pulsatille, la clematite, la jusquiame, la belladonna, la stramonée, \&c. deviennent des remèdes " ( $\left.f^{\circ} 387 \mathrm{r}\right)$. Cette glose est rigoureusement reproduite à l'impression (HDC, p. 252).

31. HDC, p. 107. 
32. HDC, p. 130.

33. HDC, p. 109.

34. HDC, p. 207-208.

35. HDC, p. 131

36. Hermann cite deux familles d'insectes distincts des papillons, quoique leurs larves puissent «filer»: les tenthrèdes ou mouches à soie, apparentés aux guêpes, et le fourmi-lion (Myrmeleontidae), dont la forme adulte ressemble à celle des libellules ou des demoiselles.

37. HDC, p. 246.

38. Des formules comme «Je me contenterai de joindre quelques observations " ( $\left.f^{\circ} 375 \mathrm{v}\right)$ sont barrées, tandis que des passages plus longs où Hermann explore une «idée qui [n'est] encore venue à aucun naturaliste » ( $\left.\mathrm{f}^{\circ} 380 \mathrm{v}\right)$ sont ignorés.

39. HDC, p. 135.

40. Lettre de Decker à la maison Levrault, Bâle, 15 juillet 1797, loc. cit.

41. HDC, p. 108

42. Sur l'approche politique du lexique de l'histoire naturelle, après 1789, voir Jean-Marc Drouin, "L'Image des sociétés d'insectes en France à l'époque de la Révolution », Revue de synthèse, IV série, tome CXIII, n³-4, juillet-décembre 1992, p. 334-335.

43. HDC, p. 81.

44. Le dossier comportant certaines feuilles isolées, il demeure possible que Hermann ait d'abord signalé sa réticence sur un tel support, désormais perdu, ou encore, qu'il en ait averti les éditeurs de manière orale.

45. HDC, p. 200

46. " Notes biographiques sur feu Jean Hermann », Bibliothèque de l'Institut, Fonds Cuvier, Ms. 3147.2, p. 14. Ce document, non daté et non signé, conservé par Cuvier pour préparer son éloge du naturaliste, pourrait avoir été composé par Jean-Frédéric Hermann, qui semble lui-même avoir caressé l'idée de réaliser ce projet de divulgation. Le dossier de Strasbourg contient en effet, de sa main, une tentative d'inventaire intitulée : « Revue des notes que mon frère a fournies à l'homme des champs de Delille et qui n'ont pas été reçues ou tronquées » (f॰ $351 \mathrm{v})$. - L'abandon du projet a sans doute évité aux deux frères un ridicule, car les notes retenues ont d'emblée paru longues à certains lecteurs. En 1800, Ginguené, qui semble le seul critique à avoir eu vent du rôle de Hermann, conclut son compte rendu du poème par un hommage ambigu : «Ce Poëme est suivi de notes fort étendues. Elles ne sont pas toutes de l'Auteur. Presque toutes celles du troisième Chant sont sur-tout d'une main étrangère. [...] On assure qu'elles ont été rédigées par le célèbre naturaliste Hermann, de Strasbourg, dont les sciences ont eu depuis à pleurer la perte. Peut-être dans une autre édition pourrait-on retrancher un peu de ce luxe scientifique. » (Pierre-Louis Ginguené, "L'Homme des champs, ou les Géorgiques françaises, par Jacques Delille», La Décade philosophique, 10 vendémiaire an IX [2 octobre 1800], p. 49, je souligne).

47. Voir Hugues Marchal, «Dire et lire l'expérience dans l'œuvre de Jacques Delille », dans A.G. Weber (dir.), Passerelles entre littérature et sciences, Paris, Classiques Garnier, 2019, p. 61-97.

48. Cet article s'inscrit dans le cadre du projet FNS « Reconstruire Delille». 


\section{RÉSUMÉS}

La poésie scientifique qui a fleuri à la fin $d u$ XVIII $^{\mathrm{e}}$ siècle autour de Jacques Delille offre un exemple frappant de l'alliance entre lettres et sciences qui demeure associée à notre image des Lumières. Mais l'examen des manuscrits laissés par le naturaliste Jean Hermann et relatifs aux notes qu'il rédigea pour L'Homme des champs de Delille permet d'aborder ce dialogue heureux comme une représentation susceptible de masquer d'intenses dissensions entre ses acteurs.

The scientific poems that flourished at the end of the 18th Century around Jacques Delille provide a striking illustration of the alliance between literature and science that we still associate to the Enlightenment. However, a close examination of the papers left by the naturalist Jean Hermann, who wrote the notes for Delille's Homme des champs, suggests that this successful dialog was largely a construction, that may have hidden intense disagreements between its various actors.

\section{INDEX}

Mots-clés : Delille (Jacques), Hermann (Jean), poésie scientifique, paratexte, critique génétique Keywords : Delille (Jacques), Hermann (Jean), scientific poetry, paratext, genetic criticism

\section{AUTEUR}

HUGUES MARCHAL

Université de Bâle 